\title{
The Evolution of the Cytochrome $c_{6}$ Family of Photosynthetic Electron Transfer Proteins
}

\author{
Barnaby Slater $\mathbb{D}^{\dagger}$, Darius Kosmützky ${ }^{\dagger}$, R. Ellen R. Nisbet $\mathbb{D}^{\ddagger, *}$, and Christopher J. Howe (D)* \\ Department of Biochemistry, University of Cambridge, United Kingdom \\ *Corresponding authors: E-mails: ellen.nisbet@nottingham.ac.uk; ch26@cam.ac.uk. \\ Accepted: 10 June 2021 \\ † These authors contributed equally to this work. \\ ${ }^{\ddagger}$ Present address: School of Biosciences, University of Nottingham, Sutton Bonington Campus, Sutton Bonington, Leicestershire, United Kingdom
}

\begin{abstract}
During photosynthesis, electrons are transferred between the cytochrome $b_{6} f$ complex and photosystem I. This is carried out by the protein plastocyanin in plant chloroplasts, or by either plastocyanin or cytochrome $c_{6}$ in many cyanobacteria and eukaryotic algal species. There are three further cytochrome $c_{6}$ homologs: cytochrome $C_{6 \mathrm{~A}}$ in plants and green algae, and cytochromes $\mathrm{C}_{6 \mathrm{~B}}$ and $\mathrm{C}_{6 \mathrm{C}}$ in cyanobacteria. The function of these proteins is unknown. Here, we present a comprehensive analysis of the evolutionary relationship between the members of the cytochrome $c_{6}$ family in photosynthetic organisms. Our phylogenetic analyses show that cytochromes $C_{6 B}$ and $C_{6 C}$ are likely to be orthologs that arose from a duplication of cytochrome $c_{6}$, but that there is no evidence for separate origins for cytochromes $C_{6 B}$ and $c_{6 C}$. We therefore propose renaming cytochrome $c_{6 C}$ as cytochrome $C_{6 B}$. We show that cytochrome $C_{6 A}$ is likely to have arisen from cytochrome $c_{6 B}$ rather than by an independent duplication of cytochrome $C_{6}$, and present evidence for an independent origin of a protein with some of the features of cytochrome $c_{6 A}$ in peridinin dinoflagellates. We conclude with a new comprehensive model of the evolution of the cytochrome $c_{6}$ family which is an integral part of understanding the function of the enigmatic cytochrome $c_{6}$ homologs.
\end{abstract}

Key words: photosynthesis, evolutionary model, phylogeny.

\section{Significance}

The cytochrome $c_{6}$ family of proteins plays an essential role in photosynthetic electron transfer, but the evolutionary relationships among the members of the family remain unclear. We show that a previously drawn distinction between cytochromes $C_{6 B}$ and $C_{6 C}$ probably reflects taxon sampling, that cytochromes $C_{6 B C}$ arose from cytochrome $C_{6}$, and that cytochrome $C_{6 A}$ subsequently arose from cytochrome $C_{6 B}$ after the divergence of the green photosynthetic lineage. These conclusions, together with a survey of the distribution of the family among eukaryotes, give us a much better understanding of the evolution of this important protein family.

\section{Introduction}

\section{The Cytochrome $c_{6}$ Family of Proteins}

Photosynthesis is one of the most important processes in the natural world and has played a vital role in shaping the planet and its atmosphere. One essential feature of oxygenic photosynthesis is the photosynthetic electron transfer chain (PETC), where the oxidation of water to generate reducing equivalents and chemical energy as ATP is driven through light energy absorption. In the plant PETC, electrons can be transferred between the cytochrome $b_{6} f$ complex and photosystem I by the copper-containing protein plastocyanin (Gross 1993). Many cyanobacteria and eukaryotic algae have an alternative electron transfer protein as a substitute for plastocyanin, the hemoprotein cytochrome $c_{6}$, which is used when copper is not readily available (Wood 1978). It is believed that cytochrome $c_{6}$ is a more ancient protein than

(C) The Author(s) 2021. Published by Oxford University Press on behalf of the Society for Molecular Biology and Evolution.

This is an Open Access article distributed under the terms of the Creative Commons Attribution License (http://creativecommons.org/licenses/by/4.0/), which permits unrestricted reuse, distribution, and reproduction in any medium, provided the original work is properly cited. 
plastocyanin, with the latter evolving after increasing atmospheric oxygen concentrations led to a decrease in the ready availability of iron in the environment (De la Rosa et al. 2002). Green plants were believed to have lost cytochrome $c_{6}$, retaining only plastocyanin (Kerfeld and Krogmann 1998).

However, in 2002 a homolog of cytochrome $c_{6}$ was found in green plants (Gupta et al. 2002; Wastl et al. 2002). This protein was subsequently named cytochrome $c_{6 A}$ (Wastl et al. 2004; fig. 1A). The sequence of cytochrome $c_{6 A}$ was found to be highly similar to that of $C_{6}$, with a major difference that cytochrome $C_{6 \mathrm{~A}}$ contains a 12-amino acid insertion in a loop region of the protein (fig. 1B). This insertion has been named the loop insertion peptide (LIP) (Howe et al. 2006), and contains two cysteines that form a disulfide bridge (Marcaida et al. 2006). Further homologs of cytochrome $c_{6 A}$ (in addition to the conventional cytochrome $c_{6}$ ) were then discovered in cyanobacteria, and named cytochromes $\mathrm{C}_{6 \mathrm{~B}}$ and $\mathrm{C}_{6 \mathrm{C}}$ (Nomura 2001; Bialek et al. 2008; fig. $1 A$ and B). These cytochromes were split into $B$ and $C$ homologs based on a phylogenetic analysis, which showed that cytochrome $C_{6 B}$ shared a more recent common ancestry with cytochrome $C_{6 A}$, and cytochrome $C_{6 C}$ shared a more recent common ancestor with cytochrome $c_{6}$ (Bialek et al. 2008).

Cytochromes $C_{6 A}, C_{6 B}$, and $C_{6 C}$ have a redox midpoint potential around $200 \mathrm{mV}$ lower than cytochrome $c_{6}$, suggesting that cytochromes $C_{6 A}, C_{6 B}$, and $C_{6 C}$ are unable to oxidize cytochrome $f$ and have a different function from cytochrome $c_{6}$ (Molina-Heredia et al. 2003; Bialek et al. 2008, 2014). This suggestion of a difference in function was supported by studies on the reaction between cytochrome $c_{6 \mathrm{~A}}$ and photosystem I in vitro and the demonstration that plastocyanin is essential in plants (Molina-Heredia et al. 2003; Weigel et al. 2003). The difference in redox midpoint potential between cytochromes $c_{6 A}$ and $c_{6}$ is proposed to be largely due to a single amino acid residue, found at position 52 in Arabidopsis thaliana cytochrome $C_{6 A}$ (Marcaida et al. 2006; fig. 1B). In the low redox midpoint potential cytochrome $\mathrm{C}_{6}$-like proteins, this residue is hydrophobic (leucine, isoleucine, or valine), with cytochrome $c_{6}$ having a conserved glutamine in the same position. Substituting the $A$. thaliana cytochrome $c_{6 A}$ valine 52 with a glutamine has been shown to increase the redox midpoint potential of the protein by around $100 \mathrm{mV}$ (Worrall et al. 2007). The function of these low redox midpoint cytochrome $c_{6}$-like proteins is currently unclear, though a role in alternative pathways in electron transfer has been proposed (Howe et al. 2016).

\section{The Current Model of Cytochrome $c_{6}$ Family Ancestry}

The current hypothesis for the evolution of the cytochrome $c_{6}$ family in photosynthetic organisms has been outlined by Howe et al. (2016) (fig. 1C). The model suggested that duplication(s) of cytochrome $c_{6}$ in an ancestral cyanobacterium led to the genesis of the low redox midpoint potential cytochromes grouped under the umbrella-term cytochrome $C_{6 A B C}$. Phylogenetic analysis by Bialek et al. (2008) suggested that at least two duplications had occurred in cyanobacteria, resulting in cytochromes $\mathrm{C}_{6 \mathrm{~B}}$ and $\mathrm{C}_{6 \mathrm{C}}$. Following primary endosymbiosis, cytochrome $c_{6}$ was lost in the green plant lineage leaving only a low redox midpoint potential sequence, cytochrome $c_{6 \mathrm{~A}}$. Secondary endosymbiosis involving the green lineage (e.g., as seen for Euglena), was believed to have failed to transfer the low redox midpoint potential cytochrome $c_{6}$. In contrast, cytochrome $c_{6 A B C}$ was believed to have been lost in the red algal and glaucophyte lineages (which contain primary plastids) sometime after the origin of the haptophytes (containing a secondary plastid; Yoon et al. 2002), which retain both cytochrome $c_{6}$ and $C_{6 A B C}$. A recent study, however, has identified a cytochrome $C_{6 B}$ homolog in the glaucophyte Cyanophora paradoxa (Kleiner et al. 2021).

\section{Aims of the Study}

With the availability of more sequence data, this study expanded the search for cytochrome $c_{6}$ family sequences in a wider range of photosynthetic taxa, both prokaryotic and eukaryotic. We particularly wished to identify whether cyanobacterial cytochrome $\mathrm{C}_{6 \mathrm{BC}}$ proteins were derived from cytochrome $c_{6}$ or vice versa, what the evolutionary relationship is between cytochrome $C_{6 \mathrm{~B}}$ and cytochrome $\mathrm{C}_{6 \mathrm{C}}$, and how widely distributed the cytochrome $C_{6 A B C}$ family is among eukaryotes.

\section{Results}

Mapping Cytochromes $C_{6}, C_{6 B}$, and $C_{6 C}$ on an Established Cyanobacterial Species Tree Shows That $C_{6 B}$ and $c_{6 C}$ Are Orthologs That Arose from a Single $c_{6}$ Gene Duplication Event

To examine the distribution of cytochromes $C_{6}, c_{6 B}$, and $C_{6 C}$ across cyanobacteria, the presence or absence of $c_{6}$ family cytochrome sequences was mapped onto a phylogenetic tree of cyanobacterial species inferred from a concatemer of conserved sequences (Walter et al. 2017; fig. 2). This phylogenetic framework represents a comprehensive phylogenomic analysis of cyanobacteria based on 31 conserved gene markers, using Maximum Likelihood (ML). Putative cytochrome $\mathrm{C}_{\mathrm{GB} / \mathrm{C}}$ sequences were found by database searching and defined as cytochrome $\mathrm{C}_{6 \mathrm{~B} / \mathrm{C}}$ if they had both an appropriately located haem-binding motif $(\mathrm{CXXCH}$; Barker and Ferguson 1999) and a valine, leucine, or isoleucine rather than glutamine at the equivalent of position 52 in A. thaliana. (The presence of valine at this position was linked to a lower redox midpoint potential relative to cytochrome $c_{6}$ [Worrall et al. 2007; Bialek et al. 2008].) 
A

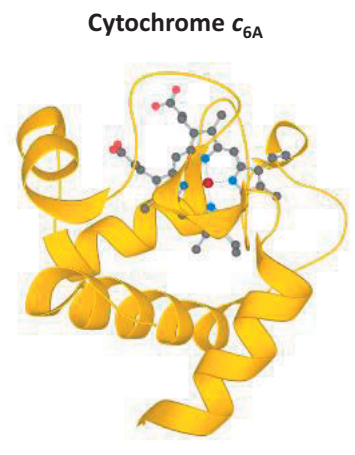

Cytochrome $c_{6}$

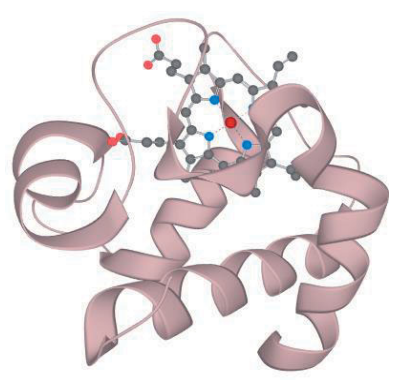

Cytochrome $c_{6 \mathrm{~B}}$

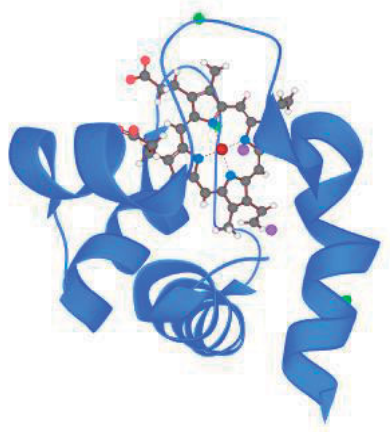

Cytochrome $c_{6 c}$

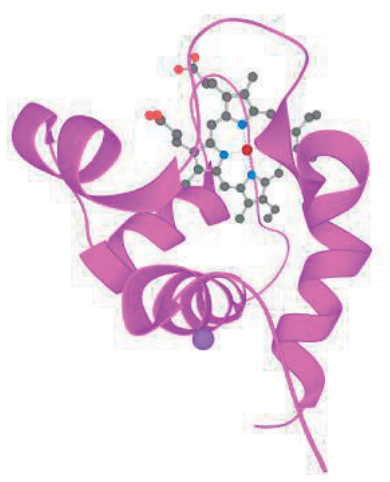

B
1
10
20
30
40
50

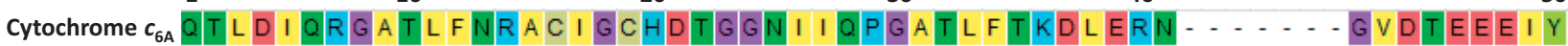
Cytochrome $c_{6}$ - - A D A A A G A V V A A NCA A C A G GNNA V M P T KTLKADALKT YLAGYKDGSKSLEEAVA Cytochrome $c_{6 B}-G E T S G E G A V L F G Q H C A G C H V N G G N I I R R G K N L K L A T L K R Q \cdots$ Cytochrome $c_{6 c}$ - ADLDQGAQ I FEAHCAGCHLNGGNIVRRGKNLKKRAMAKN - . - . GYTSVEA I A Haem-binding motif

$60 \quad 70 \quad 80 \quad 90 \quad 100$

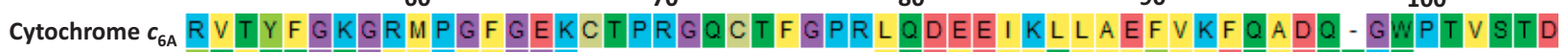

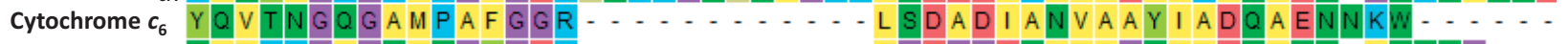

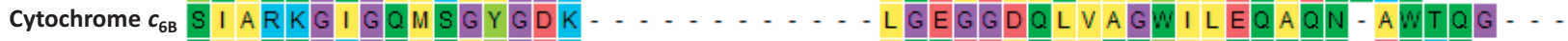

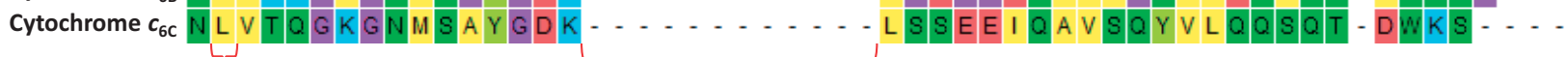

C

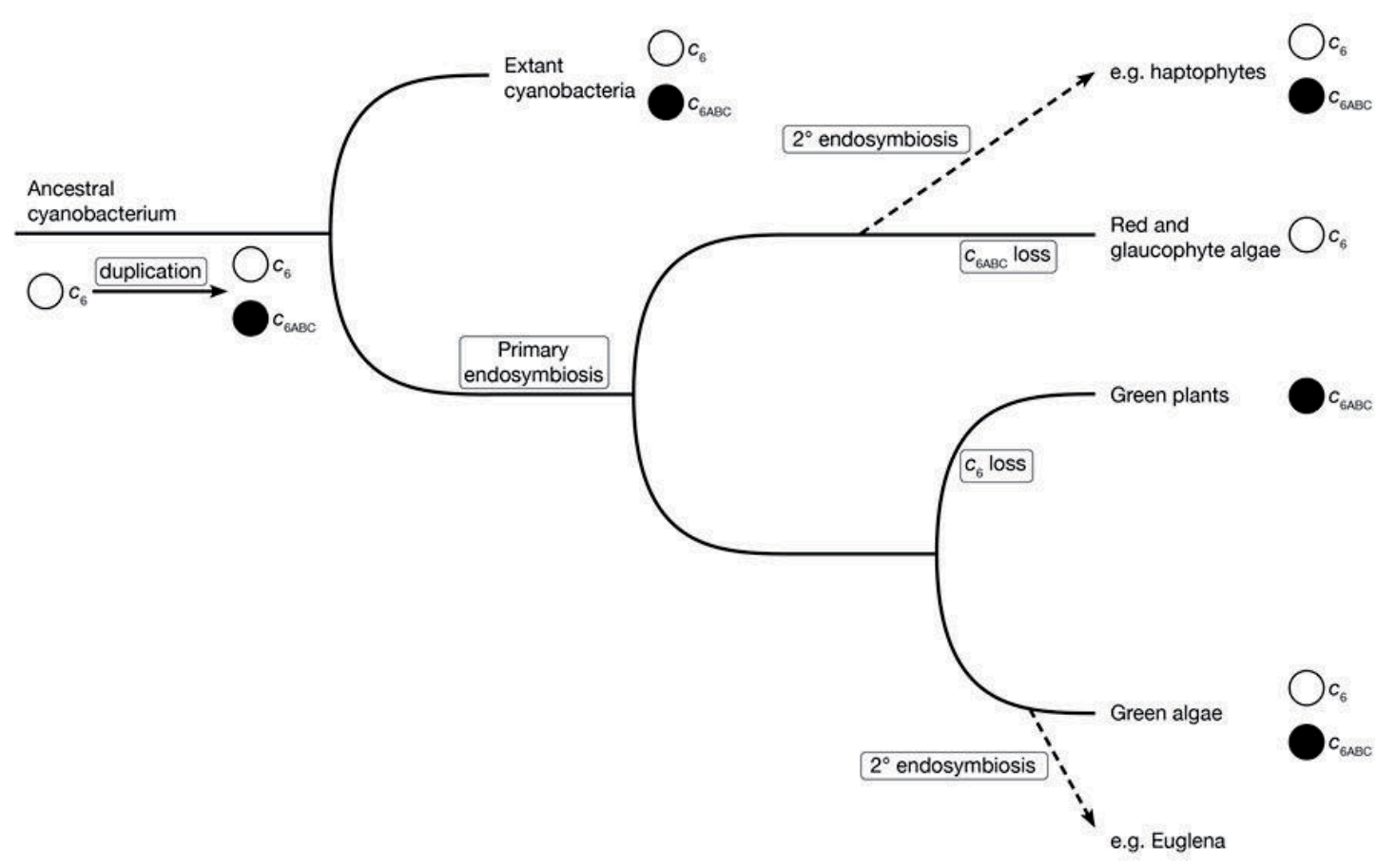

FiG. 1.-(A) X-ray crystal structures of cytochromes $c_{6 \mathrm{~A}}$ (yellow), $c_{6}$ (beige), $c_{6 \mathrm{~B}}$ (blue), and $c_{6 \mathrm{C}}$ (magenta). Secondary structure for each protein is shown in ribbon form, and the haem prosthetic groups shown in ball and stick (carbon—black, oxygen—red, nitrogen—blue, iron—deep red, and sodium— purple). (B) Protein sequence alignment of cytochromes $C_{6 A}, C_{6}, C_{6 B}$, and $C_{6 C}$ from $A$. thaliana (accession Q93VA3.1), Synechococcus sp. PCC 7002 (accession 
With this expanded sampling, the sequences identified as cytochrome $c_{6 \mathrm{~B}}$ by Bialek et al. (2008) were found exclusively in one clade, which contained cyanobacteria of the genera Prochlorococcus or Parasynechococcus, as shown in figure 2. In contrast, the sequences previously identified as cytochrome $c_{6 c}$ (Bialek et al. 2008) were widespread across the cyanobacterial species tree, but not found in the genera Prochlorococcus or Parasynechococcus. In addition, no species in the tree contained more than one cytochrome $\mathrm{C}_{6 \mathrm{~B} / \mathrm{C}}$-like sequence. These observations suggest that the prior separation observed between cytochrome $C_{6 B}$ and $c_{6 C}$ could be accounted for by taxon sampling, without needing to propose them as two separate families.

The cyanobacterial tree shows a split (labeled with a red arrow) separating taxa which have only cytochrome $c_{6}$ from those which also have a cytochrome $c_{6 \mathrm{~B}}$ or $\mathrm{C}_{6 \mathrm{C}}$ sequence. This branch point separates the basally diverging Gloeobacter violaceus PCC7421 (Criscuolo and Gribaldo 2011; Mareš et al. 2013) and a few other taxa from the rest. This distribution suggests that cytochrome $c_{6}$ appeared first, and that cytochromes $C_{6 B}$ and $C_{6 C}$ may have arisen through duplication and neofunctionalization of cytochrome $c_{6}$.

\section{A Phylogenetic Tree Using a Wider Taxon Selection Suggests a Single Origin for Cytochromes $C_{6 B}$ and $C_{6 C}$}

To investigate further the hypothesis of cytochromes $C_{6 B}$ and $c_{6 c}$ being duplicates of cytochrome $c_{6}$ with a new function, a phylogenetic tree was inferred from an alignment of cytochromes $C_{6}, C_{6 B}$, and $C_{6 C}$ sequences covering all the organisms used in two independent phylogenetic analyses of the cyanobacterial lineage (Schirrmeister et al. 2015; Walter et al. 2017). A condensed tree is shown in figure $3 A$ (the full tree can be found in supplementary fig. 1, Supplementary Material online). As the sequences are short, a Neighbor-Net analysis was also performed (fig. 3B) and showed that the data were treelike, and that tree-based phylogenetic analysis was appropriate (Huson and Bryant 2006).

The cytochromes predicted to have a low redox midpoint potential, including those assigned as cytochromes $C_{6 B}$ and $c_{6 c}$ previously, all grouped to the exclusion of the predicted cytochrome $c_{6}$ sequences in both the phylogenetic tree (bootstrap value of $84 \%$ ) and the Neighbor-Net analysis, and maintained a similar general topology to that of the cyanobacterial tree of figure 2. This distribution showed cytochrome $c_{6 \mathrm{~B}}$ as a clade derived from within the cytochrome $c_{6 C}$ clade, as with figure 2. Once again, there was no evidence of both cytochromes $C_{6 B}$ and $C_{6 C}$ within the same organism.
(Crocosphaera watsonii has been shown to have two low redox midpoint potential cytochrome $c_{6}$ sequences, but both were assigned as cytochrome $c_{6 C}$ in prior studies [Bialek et al. 2008].) These observations suggest a single origin for the cytochrome $\mathrm{C}_{6 \mathrm{BC}}$ family, and that they are orthologs rather than paralogs. It is worth noting that the bootstrap values in this tree were considerably lower than those in the tree inferred by Walter et al. (2017), which is to be expected with a larger number of taxa for sequences of short sequence length (Rokas and Carroll 2005) such as with the cytochrome $c_{6}$ family peptides. However, the tree-like appearance of the Neighbor-Net analysis inferred from the same sequence alignment suggests that phylogenetic inference is appropriate.

The neighboring open-reading frames of cytochromes $C_{6 \mathrm{~B}}$ and $c_{6 c}$ in cyanobacterial genomes were compared (data not shown). Most of the species that possess a cytochrome $C_{6 B}$ were observed to have neighboring genes coding for Nif1 domain-containing, Ycil family, or DUF3136 domaincontaining proteins, except for Prochlorococcus marinus str. MIT 9312 and MIT 9301. The most closely related species possessing a cytochrome $c_{6 c}$ had different neighboring genes from those in the cytochrome $c_{6 \mathrm{~B}}$ species. However, it is difficult to determine whether this difference in genetic neighborhoods is due to cytochromes $C_{6 B}$ and $c_{6 C}$ being paralogs, or due to the overall similarity between the genomes of species containing cytochrome $\mathrm{C}_{6 \mathrm{~B}}$, which comprise only two genera of cyanobacteria. Additionally, there was a high diversity of genetic neighborhoods amongst cytochrome $c_{6 c}$ possessing species, which represent a wider range of cyanobacteria. There was therefore no evidence from synteny to indicate that cytochromes $\mathrm{C}_{6 \mathrm{~B}}$ and $\mathrm{C}_{6 \mathrm{C}}$ are paralogs.

Taken together, there is no evidence that would support a functional differentiation between cytochromes $c_{6 B}$ and $c_{6 C}$. Cytochrome $C_{6 \mathrm{~B}}$ is found only in a clade of organisms known for a high protein substitution rate (Dufresne et al. 2005), cytochromes $C_{6 B}$ and $C_{6 C}$ both have a low redox midpoint potential, share common ancestry to the exclusion of cytochrome $c_{6}$, and are not found together in one organism. The distinction between cytochromes $C_{6 B}$ and $c_{6 C}$ does not seem to represent functional divergence, and we propose to refer to all as cytochromes $C_{6 B}$ in future.

\section{Distribution of Cytochrome $c_{6}$ Family Members across Photosynthetic Eukaryotes}

The recent sequencing of genomes and transcriptomes of a wider range of eukaryotic photosynthetic organisms allowed

O30881.1), Synechococcus WH8103 (CRY92441.1), and Synechococcus sp. PCC 7002 (accession AAN03578.1) respectively. The sequences have their putative signal peptides excluded. Amino acids are colored with yellow—hydrophobic residues, green—polar residues, beige — cysteines, blue—positively charged residues, and red-negatively charged residues, and the haem-binding motif (CXXCH), the LIP, and amino acid 52 are indicated below the alignment. Figure uses crystallography and sequence data from Marcaida et al. (2006), Bialek et al. (2009), and Zatwarnicki et al. (2014) (PDB cytochrome c6: 3DRO, c6A: 2CE0, c6B: 4KMG, c6C: 4EIE). (C) Current model of cytochrome $c_{6}$ family evolution in photosynthetic organisms. Adapted from Howe et al, (2016). 


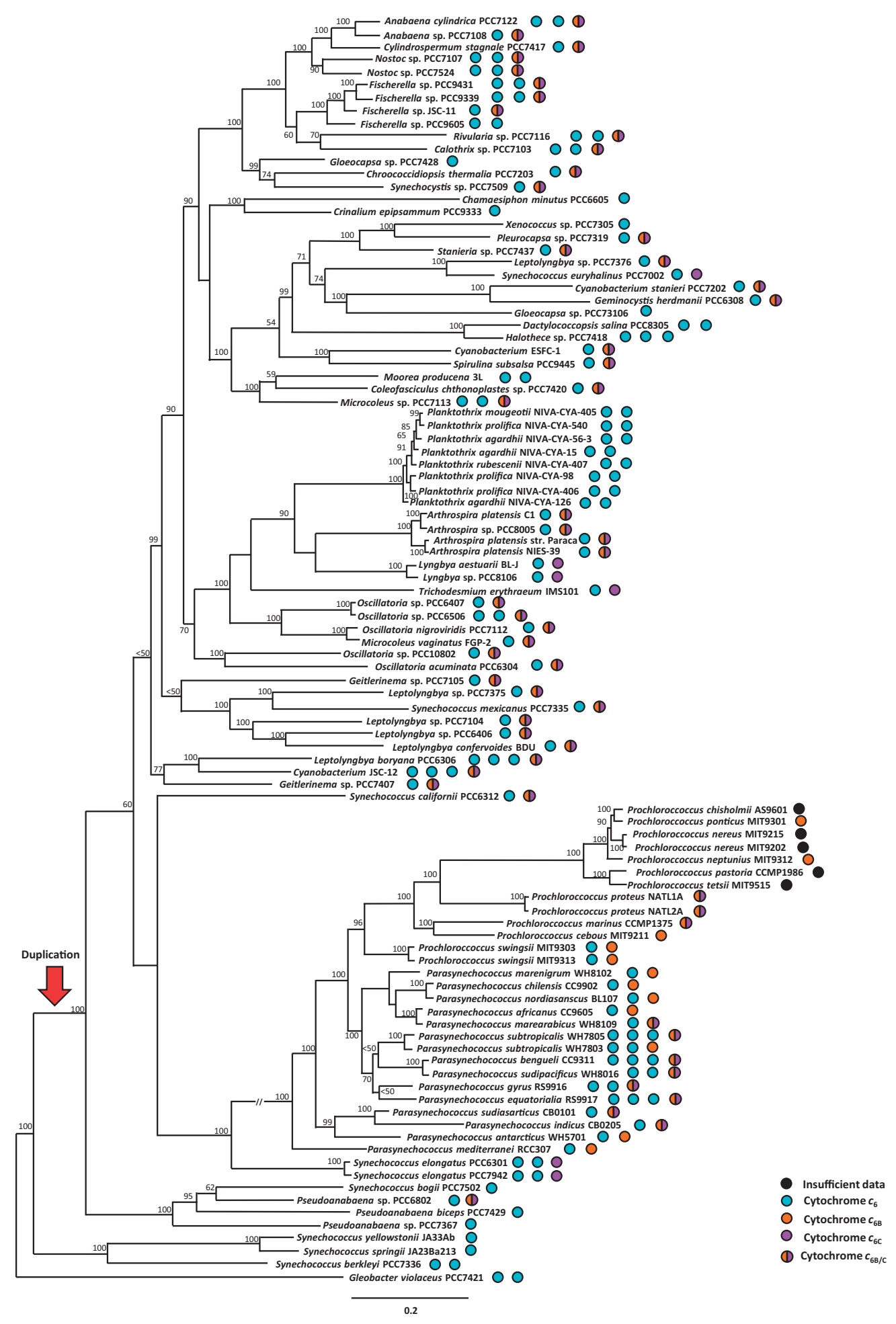

FiG. 2. - Cyanobacterial species tree with presence of cytochromes $C_{6}, C_{6 B}$, and $C_{6 C}$ mapped onto it (colored blue, orange, and magenta, respectively). Circles with both orange and magenta semicircles contain a putative low redox midpoint potential cytochrome $C_{6}$, which was not included in Bialek et al. (2008). Proteins represented by orange or purple full circles were described as $C_{6 B}$ or $C_{6 C}$, respectively by Bialek et al. (2008). Black circles represent species whose peptide or nucleotide data are not readily available to probe. Tree is reproduced from Walter et al. (2017). The potential branch where neofunctionalisation led to the evolution of cytochrome $C_{6 B}$ is indicated with a red arrow. Scale bar represents branch length. Bootstrap values were calculated as a percentage using 1,000 iterations Walter et al. (2017). Accessions of the sequences used for this figure can be found in supplementary table 1, Supplementary Material online. 
A

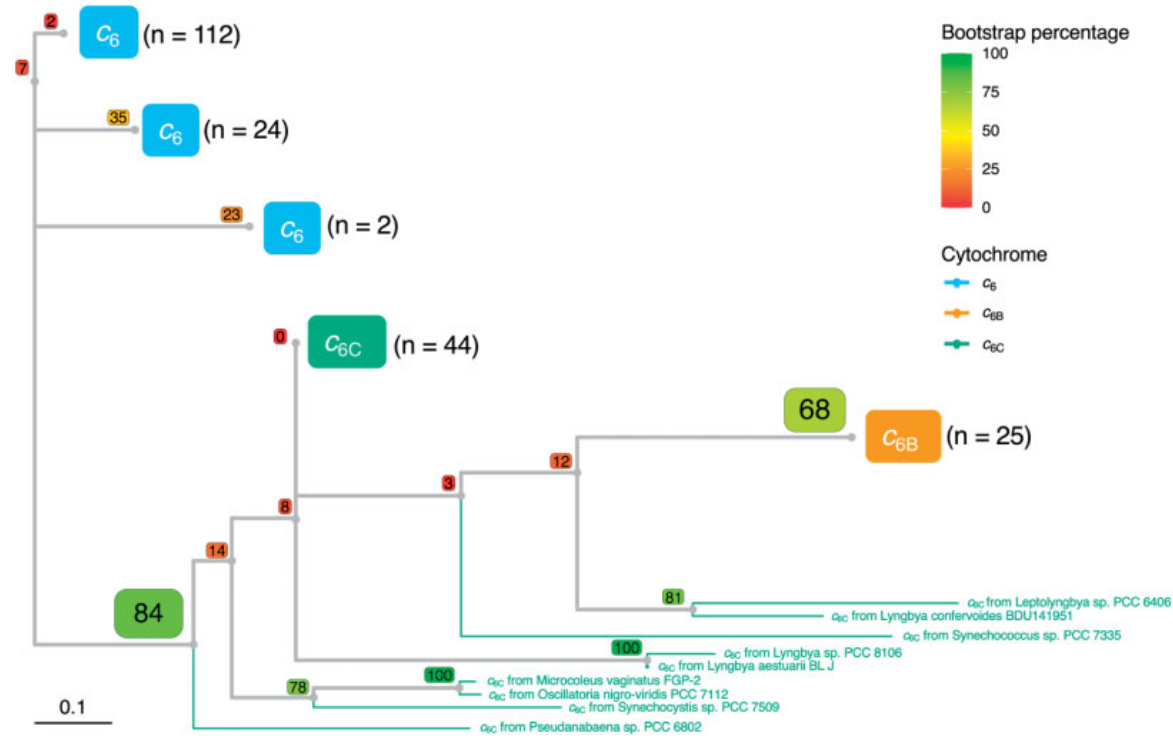

B

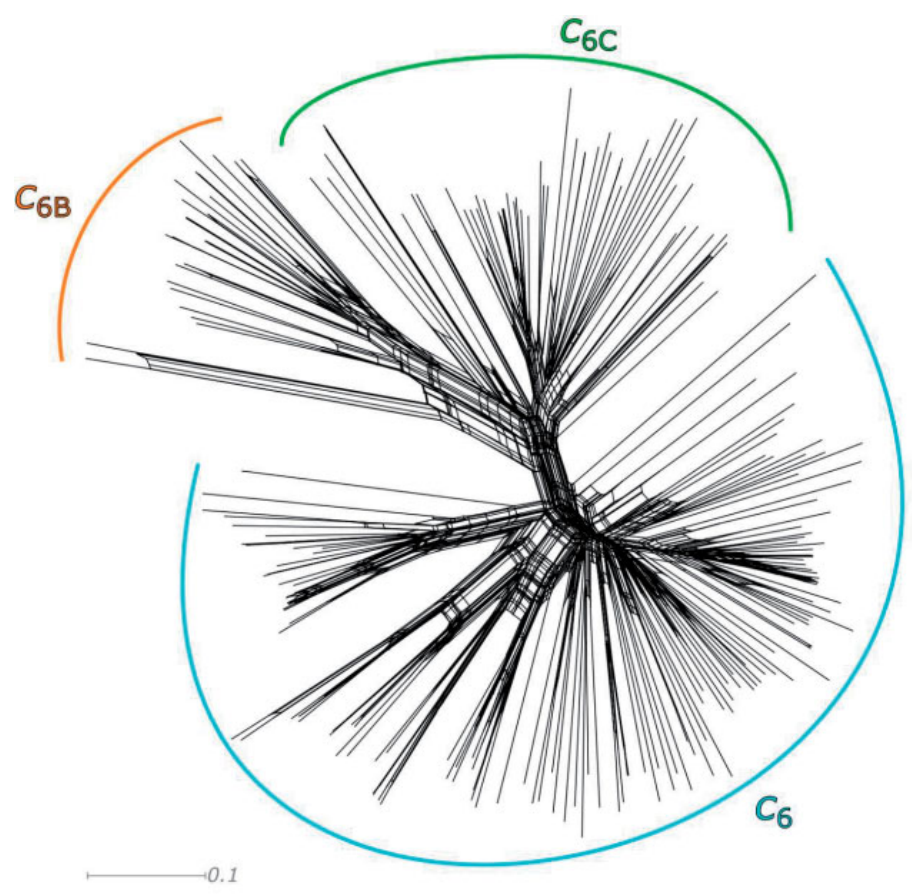

FiG. 3.-Condensed phylogenetic tree $(A)$ and Neighbor-Net splits graph $(B)$ inferred from an alignment of cytochrome $C_{6}, C_{6 B}$, and $C_{6 C}$ peptide sequences from cyanobacterial species (colored blue, orange, and green, respectively). Alignments were performed using MUSCLE algorithm and can be found in the supplementary information along with accessions for each sequence used. The phylogenetic tree was built using ML inference using a WAG model with Gamma distribution and invariable sites $(W A G+G+I)$. Bootstrap values for each branch point, using 100 iterations, are shown in colored boxes. The $n$ value next to each group represents the number of sequences found within each clade. The full tree is shown in supplementary figure 1 , Supplementary Material online and the alignment from which the tree was inferred can be found in supplementary table 2, Supplementary Material online. SplitsTree4 was used to obtain the Neighbor-Net splits graph.

for a more thorough search for $\mathrm{C}_{6}$-like cytochromes, including cytochrome $c_{6 \mathrm{~A}}$. Protein and nucleotide databases across eukaryotes were searched using BLAST for cytochrome $c_{6}$ family sequences. Sequences recovered were defined as cytochrome $c_{6 \mathrm{~A}}$ if they contained a hydrophobic residue (valine, leucine, or isoleucine) at the equivalent of position 52 , indicating a low redox midpoint potential, and an insertion containing a disulfide bridge (the LIP) in the loop region 


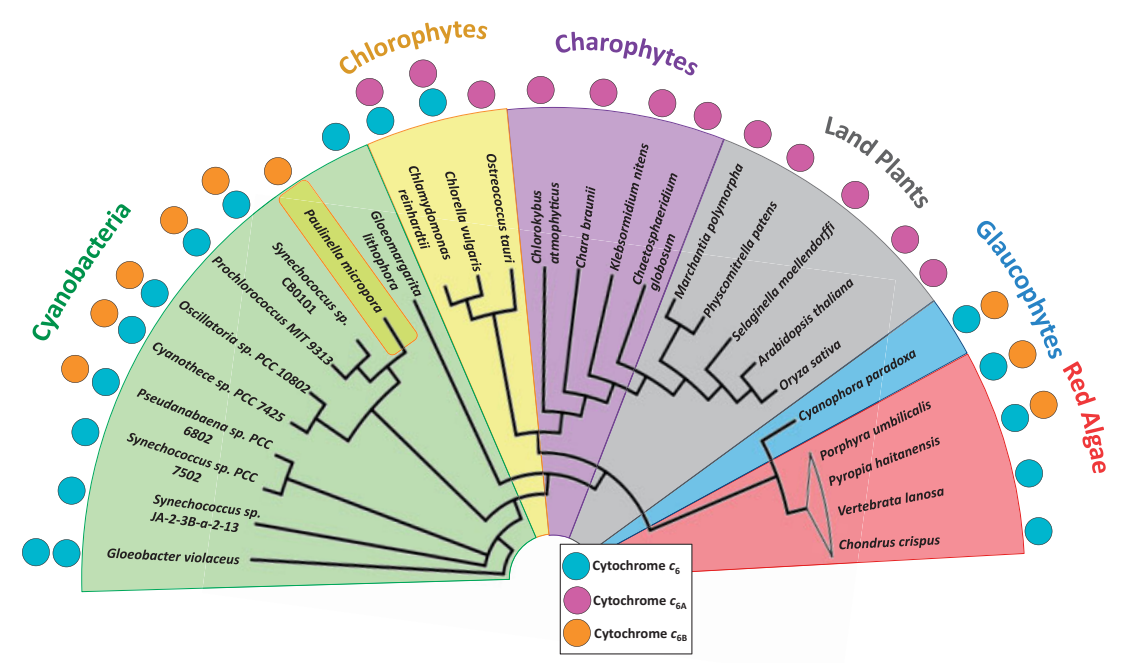

Fig. 4.-Distribution of $c_{6}$-type cytochromes across photosynthetic lineages. Presence of a colored circle adjacent to a species name indicates that a sequence of the relevant cytochrome was found in sequence database searches, with multiple copies of the same colored circle indicating a potential paralog. Paulinella chromatophora is highlighted to segregate it from the cyanobacteria. Phylogenetic tree branch lengths are not to scale. Diagram based on phylogenetic tree from Ponce-Toledo et al. (2017). Accession numbers for individual gene sequences can be found in supplementary table 3, Supplementary Material online.

compared with cytochrome $c_{6}$. Sequences recovered were defined as cytochrome $c_{6 B}$ if they contained the hydrophobic residue implying a low redox midpoint but not the LIP.

\section{Distribution of Cytochrome c6 after Primary Endosymbiosis}

The distribution of cytochromes $\mathrm{C}_{6}, \mathrm{C}_{6 \mathrm{~B}}$, and $\mathrm{C}_{6 \mathrm{~A}}$ across cyanobacteria and in eukaryotes after primary endosymbiosis was mapped onto a phylogenetic tree based on an alignment of concatemers of plastid genes and cyanobacterial homologs (fig. 4). The presence of cytochromes $c_{6}$ and $c_{6 B}$ in the glaucophyte and red algal lineages suggests that the cyanobacterium involved in the primary endosymbiosis event contained both a cytochrome $c_{6}$ and a $c_{6 B}$.

The results indicate that after primary endosymbiosis, cytochrome $c_{6 B}$ was replaced by cytochrome $c_{6 \mathrm{~A}}$ in the green plant and algal lineage. This suggests that cytochrome $c_{6 \mathrm{~A}}$ was derived from cytochrome $C_{6 B}$, possibly through an insertion of the LIP early in the green chloroplast lineage. The insertion of the LIP into an existing sequence rather than duplication and divergence is supported by the observation that no species have been found to contain both a cytochrome $C_{6 A}$ and a $C_{6 B}$ sequence. Although cytochrome $c_{6}$ was identified in some chlorophyte species, it was not identified in any charophytes or land plants, suggesting that the loss of cytochrome $c_{6}$ occurred in the ancestor to the charophyte lineage. This in turn has resulted in land plants exclusively containing cytochrome $c_{6 \mathrm{~A}}$. In contrast, the glaucophytes and many red algal species have retained the original cytochrome $c_{6}$. However, some red algal species (though not all) appeared to have lost cytochrome $\mathrm{C}_{6 \mathrm{~B}}$, for example Chondrus crispus. Finally, the eukaryotic protist Paulinella contains a cytochrome $C_{6 B}$-like sequence. This is likely to reflect the recent, independent primary endosymbiosis of a cyanobacterium that gave rise to the Paulinella chloroplast (Marin et al. 2005; Yoon et al. 2006), although the Paulinella line also appears to have lost cytochrome $c_{6}$.

\section{Distribution of Cytochrome c6 Family Members after Secondary Endosymbioses}

Many photosynthetic eukaryotes contain chloroplasts of secondary origin. We therefore searched for the presence of cytochromes $C_{6}, C_{6 B}$, and $C_{6 A}$ in these organisms. Euglena gracilis, which contains a chloroplast of secondary green origin (Turmel et al. 2009), was predicted to contain a cytochrome $c_{6 \mathrm{~A}}$ sequence in addition to cytochrome $c_{6}$ (Novák Vanclová et al. 2020). The chlorarachniophytes, a class of Rhizaria with a secondary chloroplast of green origin (Rogers et al. 2007), on the other hand only had a cytochrome $c_{6}$ sequence and no evidence of cytochrome $c_{6 \mathrm{~A}}$. This suggests that either the green algal endosymbiont of the chlorarachniophytes did not have cytochrome $c_{6 A}$ or that the gene was lost after secondary endosymbiosis.

Different organisms with a secondary red chloroplast also varied in cytochrome $c_{6}$ family gene distribution. Haptophytes, cryptomonads, and some ochrophytes, which contain a chloroplast of a red algal origin (Yoon et al. 2002), contained cytochrome $c_{6}$ and $c_{6 B}$ sequences, as expected. However, many ochrophytes and some haptophytes and cryptomonads had no evidence of $C_{6 B}$ sequences. This suggests that the red algal endosymbiont to haptophytes, cryptomonads, and ochrophytes had retained cytochrome $\mathrm{C}_{6 \mathrm{~B}}$, and that the gene was lost afterwards downstream in certain 
lineages, although widespread lateral transfer cannot be excluded.

The situation in dinoflagellate algae is complex. The peridinin dinoflagellates contain a chloroplast of secondary red origin (Dorrell and Howe 2015). Two peridinin dinoflagellates (Amphidinium carterae and Symbiodinium microadriaticum) contain a cytochrome $c_{6}$ sequence and what appeared to be a cytochrome $c_{6 \mathrm{~A}}$ sequence. In contrast, Karlodinium veneficum, a fucoxanthin dinoflagellate (which obtained its chloroplast via loss of the red algal chloroplast and serial endosymbiosis of a haptophyte [Dorrell and Howe 2015; Klinger et al. 2018]), contains a cytochrome $c_{6}$ and cytochrome $C_{6 B}$. The existence of a cytochrome $C_{6 B}$ in the Karlodinium lineage, whose chloroplast is of red algal origin, is not surprising. However, the peridinin dinoflagellate chloroplast is also of red algal origin, so the apparent existence of cytochrome $C_{6 \mathrm{~A}}$ in this lineage is unexpected.

The sequences resembling cytochrome $c_{6 \mathrm{~A}}$ in peridinin dinoflagellate algae were compared with those of other cytochrome $c_{6 \mathrm{~A}}$ proteins (fig. 5). This revealed that the dinoflagellate sequences show some sequence similarity with the cytochromes $C_{6 \mathrm{~A}}$ from the green chloroplast lineage. The LIP insertion, however, shows very little sequence similarity between dinoflagellates and green plants, except for the two characteristic cysteine residues. It should also be noted that the dinoflagellate sequences are longer than the cytochrome $C_{6 A}$ sequences from green plants, with the first position of each of the dinoflagellate sequences in figure 5 being residues 123 and 50 for $A$. carterae and S. microadriaticum, respectively. These observations suggest that the putative dinoflagellate cytochrome $c_{6 \mathrm{~A}}$ sequences have a functional similarity to cytochrome $c_{6 \mathrm{~A}}$ from green plants, but are likely to have an independent origin.

A summary of the photosynthetic eukaryotes and the presence of each cytochrome $c_{6}$ family sequence discovered can be found in table 1. Taxon IDs of clades searched are in supplementary table 4, Supplementary Material online, and accession numbers of the sequences found are in supplementary table 1, Supplementary Material online.

Cytochrome $c_{6 \mathrm{~A}}$ Arose from Cytochrome $\mathrm{C}_{6 \mathrm{~B}}$ Rather Than Directly from Cytochrome $c_{6}$

To test if cytochrome $c_{6 \mathrm{~A}}$ (non-dinoflagellate) arose from cytochrome $C_{6 B}$, rather than by independent modification of a cytochrome $c_{6}$, a phylogenetic tree was inferred using cytochrome $C_{6 \mathrm{~A}}$ sequences from eukaryotic algae and green plants, together with cytochromes $C_{6}$ and $C_{6 B}$ from a wide range of cyanobacteria (fig. 6 shows a condensed version of this tree, and the full tree can be found in supplementary fig. 2 , Supplementary Material online). Cytochromes $C_{6 \mathrm{~A}}$ and $\mathrm{C}_{6 \mathrm{~B}}$ grouped together to the exclusion of cytochrome $c_{6}$ (bootstrap value of 75), suggesting that cytochrome $C_{6 A}$ shares most recent common ancestry with cytochrome $\mathrm{C}_{6 \mathrm{~B}}$. This supports the conclusion above (fig. 4) that cytochrome $C_{6 \mathrm{~A}}$ was derived from cytochrome $c_{6 \mathrm{~B}}$ through an insertion event in the loop region, rather than independently of cytochrome $\mathrm{C}_{6 \mathrm{~B}}$. Once again, the bootstrap values in the tree were considerably lower than those of the species tree established by Walter et al. (2017), but this is to be expected as the $c_{6}$ family cytochrome sequences are short. (A Neighbor-Net splits graph for this alignment was also constructed (not shown) but was less clearly resolved and did not give any additional information.)

\section{Discussion}

\section{Cytochromes $\mathrm{C}_{6 \mathrm{~B}}$ and $\mathrm{C}_{6 \mathrm{C}}$ Are Orthologs}

The original differentiation of cytochromes $C_{6 \mathrm{~B}}$ and $\mathrm{C}_{6 \mathrm{C}}$ was based on the sequence data available at the time (Bialek et al. 2008). However, now that more genomic sequence data are available, cytochrome $c_{6}$ family sequences from a larger range of taxa can be analyzed. Our analysis indicates that the distinction between cytochromes $\mathrm{C}_{6 \mathrm{~B}}$ and $\mathrm{c}_{6 \mathrm{C}}$ can be accounted for by taxon sampling rather than differences in function. (Although cytochromes $C_{6 B}$ and $C_{6 C}$ lie on opposite sides of the root of the cytochrome $c_{6}$ family in the tree of Bialek et al. [2008], the placing of the root should be viewed with caution given that it depends on other c-type cytochromes of very different function from the cytochrome $c_{\sigma}$ family.) In addition, as the crystal structures, surface charge distribution, and redox midpoint potentials of cytochromes $C_{6 B}$ and $C_{6 C}$ are notably similar (Zatwarnicki et al. 2014; fig. 1A and B), it seems likely that cytochromes $C_{6 B}$ and $C_{6 C}$ perform a similar function and are thus orthologs.

\section{Two Independent Origins of $\mathrm{C}_{6 \mathrm{~A}}$}

Although the presence of cytochrome $c_{6 \mathrm{AA}}$ in plants and green algae has been known for time, the presence of cytochrome $c_{6 \mathrm{~A}}$ in peridinin dinoflagellates was unexpected. Dinoflagellates contain chloroplasts of secondary or tertiary origin, depending on species. The chloroplast found in S. microadriaticum and $A$. carterae contains peridinin, and is believed to represent the ancestral dinoflagellate chloroplast. This chloroplast was most likely obtained through secondary endosymbiosis of red algae (Dorrell and Howe 2015). Therefore, these species might be expected to contain a cytochrome $c_{6 B}$. Instead, the peridinin dinoflagellates contain a cytochrome $c_{6 A}$-like sequence. Two hypotheses for this are 1) the result of lateral gene transfer from an organism with cytochrome $c_{6 \mathrm{~A}}$ and the loss of the cytochrome $c_{6 \mathrm{~B}}$ or 2) the insertion of a LIP-like sequence into an existing cytochrome $\mathrm{C}_{6 \mathrm{~B}}$ sequence. Although lateral gene transfer to dinoflagellates from other organisms has been well documented (Takishita et al. 2003; Hackett et al. 2005; Chan et al. 2012; Wisecaver et al. 2013), and it is difficult to exclude conclusively lateral transfer of cytochrome $c_{6 \mathrm{~A}}$ into the dinoflagellates, the low sequence similarity between the dinoflagellate $c_{6 A}$ and those from the green 


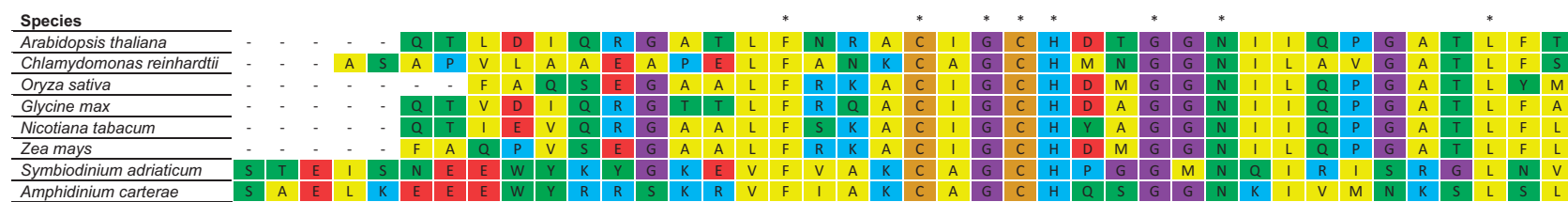

Amphidinium carterae
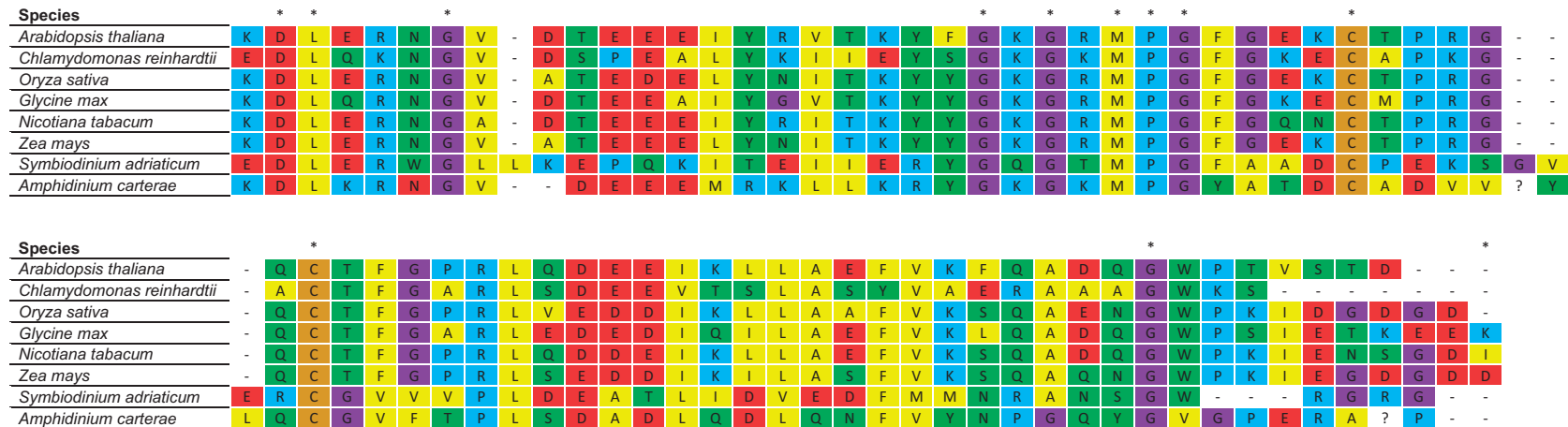

FIG. 5.-MUSCLE alignment of cytochrome $C_{6 A}$ from green eukaryotic lineages and the putative cytochrome $C_{6 A}$ sequences from the dinoflagellates $S$. microadriaticum and A. carterae. Amino acids are colored such that yellow—hydrophobic residues, green — polar residues, blue — positively charged residues, beige — cysteines, purple — glycines, light green—tyrosine, and red—negatively charged residues. Dashes indicate inserted gaps. Asterisks represent conserved residues. The sequences of the mature dinoflagellate proteins have been cut for a clearer depiction of the alignment, with the $S$. microadriaticum sequence beginning at amino acid 50 and the $A$. carterae sequence beginning at amino acid 123. Accessions used: $A$. thaliana (AT5G45040.1), C. reinhardtii (XP_001692119.1), O. sativa (EAZ04378.1), G. max (KRH50430.1), N. tabacum (XP_016489567.1), Z. mays (ACN28933.1), S. microadriaticum (OLP91854.1), and A. carterae (CF065358.1).

Table 1

The Presence or Absence of Cytochrome $c_{6}$ Family Members across Photosynthetic Eukaryotes

\begin{tabular}{|c|c|c|c|c|c|}
\hline \multirow[t]{2}{*}{ Supergroup } & \multirow[t]{2}{*}{ Clade $^{a}$} & \multirow[t]{2}{*}{ Plastid } & \multicolumn{3}{|c|}{ Cytochrome } \\
\hline & & & $c_{6}^{b}$ & $c_{6 A}^{b}$ & $c_{6 B}^{b}$ \\
\hline Glaucophyta & Glaucophyta $(n=1)$ & Primary & $\checkmark(1)$ & $\mathrm{O}$ & $\checkmark(1)$ \\
\hline Rhodophyta & Rhodophyta $(n=190)$ & Primary & $\checkmark(190)$ & $\mathrm{O}$ & $\checkmark(4)$ \\
\hline Viridiplantae & Chlorophyta $(n=42)$ & Primary & $\checkmark(32)$ & $\checkmark(34)$ & $\mathrm{O}$ \\
\hline Viridiplantae & Streptophyta $(n=217)$ & Primary & $\mathrm{O}$ & $\checkmark(215)$ & 0 \\
\hline \multirow[t]{2}{*}{ SAR—Rhizaria } & Paulinella $(n=3)$ & Primary cyanobacteria & $\mathrm{O}$ & $\mathrm{O}$ & $\checkmark(3)$ \\
\hline & $\begin{array}{l}\text { Chlorarachniophyta } \\
\qquad(n=2)\end{array}$ & Secondary green & $\checkmark(2)$ & $\mathrm{O}$ & $\mathrm{O}$ \\
\hline Cryptomonads & Cryptomonads $(n=7)$ & Secondary (rhodophyta) & $\checkmark(2)$ & $\mathrm{O}$ & $\checkmark(1)$ \\
\hline Haptista & Haptophyta $(n=6)$ & Secondary (rhodophyta) & $\checkmark(6)$ & $\mathrm{O}$ & $\checkmark(3)$ \\
\hline SAR-Heterokonta & Ochrophyta $(n=80)$ & Secondary (rhodophyta) & $\checkmark(78)$ & $\mathrm{O}$ & $\checkmark(2)$ \\
\hline SAR_Alveolata & Dinoflagellata $(n=7)$ & $\begin{array}{l}\text { Secondary (rhodophyta)/ } \\
\text { Tertiary (haptophyta) }\end{array}$ & $\checkmark(6)$ & $\checkmark(2)$ & $\checkmark(1)$ \\
\hline Discoba-Euglenozoa & Euglenids $(n=2)$ & Secondary (chlorophyta) & $\checkmark(2)$ & $\checkmark(1)$ & 0 \\
\hline
\end{tabular}

${ }^{a}$ Where $n$ represents the number of organisms searched.

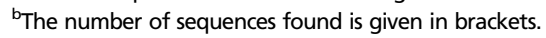

plant lineage would suggest an independent LIP insertion into cytochrome $c_{6 B}$ in dinoflagellates is more likely.

\section{The Current Model of Cytochrome c 6 Family Ancestry}

The analysis of the cytochromes $C_{6}, C_{6 A}$, and $C_{6 B}$ in this study provides a revised evolutionary model for cytochrome $C$ homologs consistent with more extensive taxon sampling (fig. 7). As more anciently diverged cyanobacterial species such as Gloeobacter appear to contain cytochrome $c_{6}$ exclusively, this suggests that the low redox midpoint potential cytochromes are more recent than cytochrome $c_{6}$. A duplication of cytochrome $c_{6}$, followed by point mutations that lowered the redox midpoint potential, led to the evolution of cytochrome $C_{6 B}$. This is supported by the presence of both cytochromes $C_{6}$ and $c_{6 B}$ in most extant cyanobacteria today. 


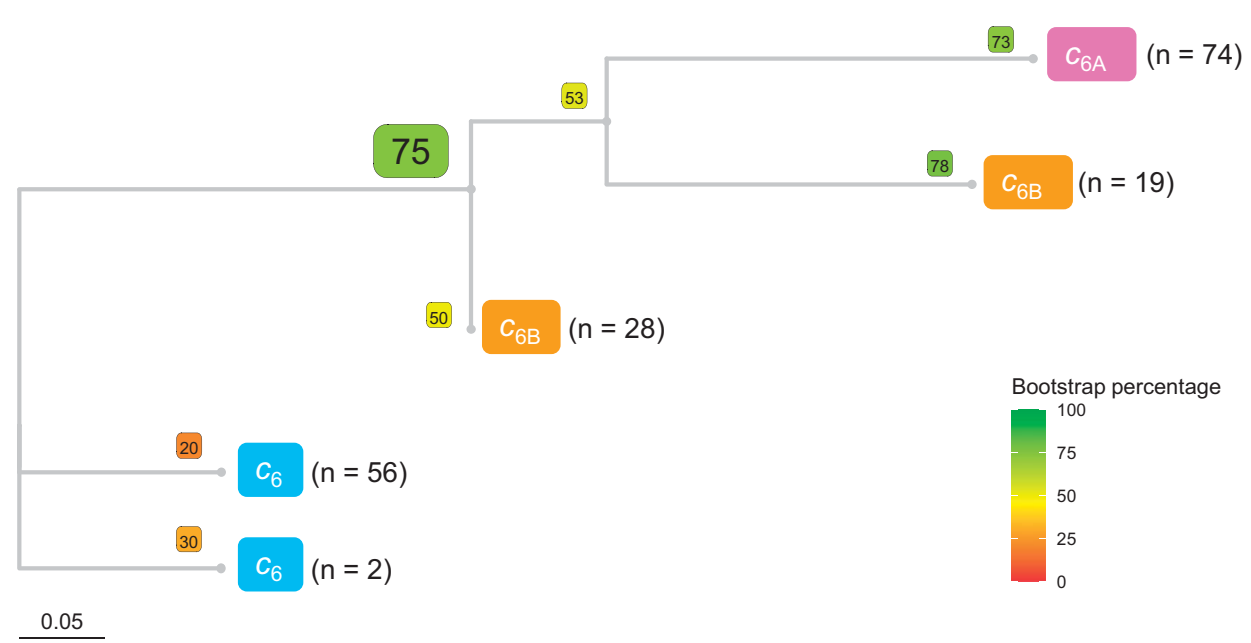

FiG. 6.-Condensed phylogenetic tree inferred from an alignment of cytochrome $c_{6}, c_{6 A}$, and $C_{6 B}$ peptide sequences (colored blue, pink, and orange, respectively) from eukaryotic algae, green plants, and cyanobacteria. Alignments were performed using MUSCLE algorithm and can be found in the supplementary information along with accessions for each sequence used. The tree was built using ML inference using a WAG model with Gamma distribution and invariable sites (WAG $+\mathrm{G}+\mathrm{I}$ ). Bootstrap values for each branch point, using 100 iterations, are shown in colored boxes. The full tree is shown in supplementary figure 2, Supplementary Material online and the alignment from which the tree was inferred can be found in supplementary table 5, Supplementary Material online.

At primary endosymbiosis, giving rise to the red, green, and glaucophyte chloroplasts, the genes were transferred to photosynthetic eukaryotes.

Red algal lineages and the glaucophytes contain cytochromes $C_{6}$ and $C_{6 B}$, although some red algal species have lost cytochrome $C_{6 B}$. In the green chloroplast lineages, cytochrome $c_{6 B}$ was replaced by cytochrome $c_{6 A}$. This was probably due to an insertion of the LIP into cytochrome $C_{6 B}$, as cytochrome $C_{6 \mathrm{~A}}$ is monophyletic within cytochrome $C_{6 \mathrm{~B}}$ (fig. 6). In many chlorophyte species, both cytochromes $C_{6}$ and $c_{6 A}$ are present. In the charophytes, ancestors to the green land plants, cytochrome $c_{6}$ was lost. In consequence, land plants contain only a cytochrome $c_{6 \mathrm{~A}}$.

Organisms containing chloroplasts of secondary origin appear have inherited their cytochrome $c_{6}$ family genes from the relevant endosymbiont. The haptophytes obtained both cytochromes $c_{6}$ and $c_{6 B}$ from the red algal chloroplast, and these genes were transferred to the fucoxanthin dinoflagellates following serial endosymbiosis. In contrast, the peridinin dinoflagellates, containing chloroplasts of secondary red origin, probably converted the cytochrome $\mathrm{C}_{6 \mathrm{~B}}$ into a cytochrome $C_{6 A}$-like protein through the insertion of a novel LIP. With green plastid secondary endosymbiosis, genes for both cytochromes $c_{6}$ and $C_{6 A}$ were passed to the euglenids (Novák Vanclová et al. 2020).

Overall, it is clear that the low potential cytochrome $C_{6 A B}$ family is widely, but not universally, present among oxygenic photosynthetic organisms. It is unlikely to be essential under all conditions, but there is no obvious environmental feature common to those organisms that retain a member of the family. The function of the cytochrome $c_{6 A B}$ family remains to be determined.

\section{Materials and Methods}

\section{Construction of Phylogenetic Trees}

A cytochrome $C_{6 B}$ sequence (BAD79758.1) was used for searching the "non-redundant protein sequences $(n r)$ " database with the NCBI BLASTp algorithm, limiting the results to the organisms used in two independent phylogenetic analyses of the cyanobacterial lineage (Schirrmeister et al. 2015; Walter et al. 2017). A cytochrome $c_{6 A}$ protein sequence (AED95193.1) was used to search the $n r$ protein database and the "nucleotide collection (nr/nt)" nucleic acid database with the BLASTp and tBLASTn algorithms respectively, limiting the results to the orders used in a phylogenetic analysis of green plants (Ruhfel et al. 2014). The resulting sequences of both searches were downloaded from the NCBI BLAST result page.

The retrieved peptide sequences were imported into MEGA7 (Kumar et al. 2016). Sequences were deleted from the selection if they were too short or too long to be a valid cytochrome $c_{6(\mathrm{AB})}$ sequence (less than 80 and more than 200 amino acids before $\mathrm{N}$-terminal targeting peptide trimming) or did not have a $\mathrm{CxxCH}$ haem binding motif. The sequences were aligned using the MUSCLE algorithm with UPGMA clustering method and a gap-opening penalty of -2.9 and no gap extension penalty. Subsequently the putative signal peptides were trimmed from the sequences.

The WAG model with gamma distribution and invariable sites (WAG + G + I) (Whelan and Goldman 2001) was determined to be optimal for tree inference with maximum likelihood (ML) by the "Find Best DNA/Protein Models (ML)" tool in MEGA7 and was thus used in the algorithm parameters for tree inference. Statistical testing was performed using the 


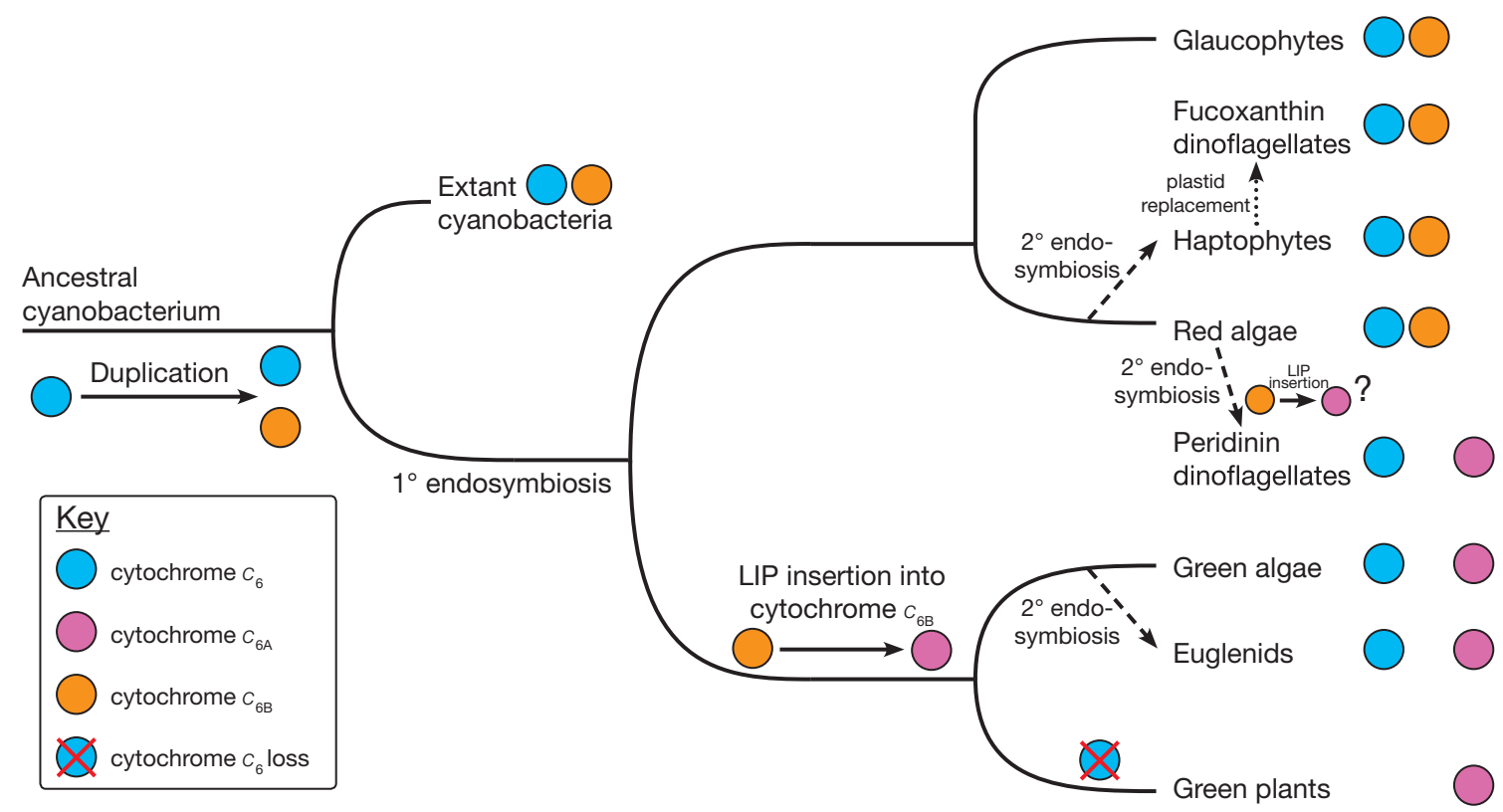

FiG. 7.-Updated ancestry model for the cytochrome $c_{6}$ family in photosynthetic organisms.

bootstrap method with 100 iterations. The final trees were visualized using Treeio in RStudio (Wang et al. 2020). The accessions of the sequences used for inference of phylogenetic trees can be found in supplementary tables 2 and 5, Supplementary Material online.

The same aligned sequences were imported into SplitsTree4 (Huson and Bryant 2006), and the software was used to build a Neighbor-Net splits graph.

\section{Database Queries for Peptide Sequences}

Searches for protein sequences homologous to the cytochrome $c_{6}$ family, or nucleotide sequences encoding them, were performed using NCBI BLAST both in BLASTp and tBLASTn (https://www.ncbi.nlm.nih.gov/). The cytochrome $c_{6}$ $C_{6 A}, C_{6 B}$, and $C_{6 C}$ peptide sequences (without targeting) used in both BLASTp and tBLASTn searches were from accessions ALJ67080.1, AED95193.1, AAP99622.1, and ACB00369.1, respectively. For BLASTp searches, the database searched was "Non-redundant protein sequences (nr)" with default parameters. For tBLASTn searches, the databases searched were "nucleotide collection (nr/nt)," "Whole-genome shotgun contigs (wgs)," and "Expressed sequence tags (est)" with default parameters. Having identified a putative cytochrome $c_{6}$ sequence, each organism that provided a query sequence was searched again to confirm the query as the best hit.

\section{Supplementary Material}

Supplementary data are available at Genome Biology and Evolution online.

\section{Acknowledgments}

This work was supported by a Biotechnology and Biological Sciences Research Council DTP PhD studentship (BB/ M011194/1 to B.S.); the German Academic Scholarship, the Gates Cambridge Trust and the Benn W Levy Trust to D. K.; and the Gordon and Betty Moore Foundation (GBMF4976, doi: https://doi.org/10.37807/GBMF4976 and GBMF9358, doi: https://doi.org/10.37807/GBMF9358 to R.E.R.N. and C.J.H.).

\section{Data Availability}

The data underlying this article are available at the NCBI. References for individual genes are given in the article and/ or in the Supplementary Material online. All alignments are provided in the supplementary Material online.

\section{Literature Cited}

Barker PD, Ferguson SJ. 1999. Still a puzzle: why is haem covalently attached in c-type cytochromes? Structure 7(12):R281-R290.

Bialek W, Krzyda S, Jaskolski M, Szczepaniak A. 2009. Atomic-resolution structure of reduced cyanobacterial cytochrome $c_{6}$ with an unusual sequence insertion. Febs J. 276(16):4426-4436.

Bialek W, Nelson M, Tamiola K, Kallas T, Szczepaniak A. 2008. Deeply branching $\mathrm{C}_{6}$-like cytochromes of cyanobacteria. Biochemistry 47(20):5515-5522.

Bialek W, et al. 2014. Insights into the relationship between the haembinding pocket and the redox potential of $c_{6}$ cytochromes: four atomic resolution structures of $c_{6}$ and $c_{6}$-like proteins from Synechococcus sp. PCC 7002. Acta Crystallogr D Biol Crystallogr. 70(Pt 11):2823-2832.

Chan CX, et al. 2012. Analysis of Alexandrium tamarense (dinophyceae) genes reveals the complex evolutionary history of a microbial eukaryote. J Phycol. 48(5):1130-1142. 
Criscuolo A, Gribaldo S. 2011. Large-Scale phylogenomic analyses indicate a deep origin of primary plastids within cyanobacteria. Mol Biol Evol. 28(11):3019-3032.

De la Rosa MA, et al. 2002. An evolutionary analysis of the reaction mechanisms of photosystem I reduction by cytochrome $c_{6}$ and plastocyanin. Bioelectrochemistry 55(1-2):41-45.

Dorrell RG, Howe CJ. 2015. Integration of plastids with their hosts: lessons learned from dinoflagellates. Proc Natl Acad Sci U S A. 112(33):10247-10254.

Dufresne A, Garczarek L, Partensky F. 2005. Accelerated evolution associated with genome reduction in a free-living prokaryote. Genome Biol. 6(2):R14.

Gross EL. 1993. Plastocyanin: structure and function. Photosynth Res. 37(2):103-116.

Gupta R, He Z, Luan S. 2002. Functional relationship of cytochrome $c_{6}$ and plastocyanin in Arabidopsis. Nature 417(6888):567-571.

Hackett JD, et al. 2005. Insights into a dinoflagellate genome through expressed sequence tag analysis. BMC Genomics. 6(1):80.

Howe CJ, Nimmo RH, Barbrook AC, Bendall DS. 2016. Cytochrome $c_{6 A}$ of chloroplasts. In: Cramer WA, and Kallas T, editors. Cytochrome complexes: evolution, structures, energy transduction, and signaling. Dordrecht: Springer. p. 701-712.

Howe CJ, Schlarb-Ridley BG, Wastl J, Purton S, Bendall DS. 2006. The novel cytochrome $C_{6}$ of chloroplasts: a case of evolutionary bricolage? J Exp Bot. 57(1):13-22.

Huson DH, Bryant D. 2006. Application of phylogenetic networks in evolutionary studies. Mol Biol Evol. 23(2):254-267.

Kerfeld CA, Krogmann DW. 1998. Photosynthetic cytochromes c in Cyanobacteria, algae, and plants. Annu Rev Plant Physiol Plant Mol Biol. 49:397-425.

Kleiner $\mathrm{FH}$, Vesteg M, Steiner JM. 2021. An ancient glaucophyte $\mathrm{c}_{6}$-like cytochrome related to higher plant cytochrome $c_{6 \mathrm{~A}}$ is imported into muroplasts. J Cell Sci. 134(9):jcs255901.

Klinger CM, et al. 2018. Plastid transcript editing across dinoflagellate lineages shows lineage-specific application but conserved trends. Genome Biol Evol. 10(4):1019-1038.

Kumar S, Stecher G, Tamura K. 2016. MEGA7: molecular evolutionary genetics analysis version 7.0 for bigger datasets. Mol Biol Evol. 33(7):1870-1874.

Marcaida MJ, et al. 2006. Structure of cytochrome $c_{6 A}$, a novel dithiocytochrome of Arabidopsis thaliana, and its reactivity with plastocyanin: implications for function. J Mol Biol. 360(5):968-977.

Mareš J, et al. 2013. The primitive thylakoid-less cyanobacterium Gloeobacter is a common rock-dwelling organism. PLoS One. 8(6):e66323.

Marin B, Nowack ECM, Melkonian M. 2005. A plastid in the making: evidence for a second primary endosymbiosis. Protist 156(4):425-432.

Molina-Heredia FP, et al. 2003. A new function for an old cytochrome? Nature 424(6944):33-34.

Nomura CT. 2001. Electron transport of Synechococcus sp. PCC 7002 [dissertation]. Pennsylvania: Pennsylvania State University.

Novák Vanclová AMG, et al. 2020. Metabolic quirks and the colourful history of the Euglena gracilis secondary plastid. New Phytol. 225(4):1578-1592.

Ponce-Toledo Rl, et al. 2017. An early-branching freshwater cyanobacterium at the origin of plastids. Curr Biol. 27(3):386-391.
Rogers MB, Gilson PR, Su V, McFadden GI, Keeling PJ. 2007. The complete chloroplast genome of the chlorarachniophyte Bigelowiella natans: evidence for independent origins of chlorarachniophyte and euglenid secondary endosymbionts. Mol Biol Evol. 24(1):54-62.

Rokas A, Carroll SB. 2005. More genes or more taxa? The relative contribution of gene number and taxon number to phylogenetic accuracy. Mol Biol Evol. 22(5):1337-1344.

Ruhfel BR, Gitzendanner MA, Soltis PS, Soltis DE, Burleigh JG. 2014. From algae to angiosperms-inferring the phylogeny of green plants (Viridiplantae) from 360 plastid genomes. BMC Evol Biol. 14:23.

Schirrmeister BE, Gugger M, Donoghue PCJ. 2015. Cyanobacteria and the great oxidation event: evidence from genes and fossils. Palaeontology 58(5):769-785

Takishita K, Ishida Kl, Maruyama T. 2003. An enigmatic GAPDH gene in the symbiotic dinoflagellate genus Symbiodinium and its related species (the order Suessiales): possible lateral gene transfer between two eukaryotic algae, dinoflagellate and euglenophyte. Protist 154(34):443-454.

Turmel M, Gagnon MC, O'Kelly CJ, Otis C, Lemieux C. 2009. The chloroplast genomes of the green algae Pyramimonas, Monomastix, and Pycnococcus shed new light on the evolutionary history of prasinophytes and the origin of the secondary chloroplasts of euglenids'. Mol Biol Evol. 26(3):631-648.

Walter JM, et al. 2017. Ecogenomics and taxonomy of Cyanobacteria phylum. Front Microbiol. 8:2132.

Wang LG, et al. 2020. treeio: an $R$ package for phylogenetic tree input and output with richly annotated and associated data. Mol Biol Evol. 37(2):599-603.

Wastl J, Bendall DS, Howe CJ. 2002. Higher plants contain a modified cytochrome c(6). Trends Plant Sci. 7(6):244-245

Wastl J, Purton S, Bendall DS, Howe CJ. 2004. Two forms of cytochrome $C_{6}$ in a single eukaryote. Trends Plant Sci. 9(10):474-476.

Weigel $\mathrm{M}$, et al. 2003. Plastocyanin is indispensable for photosynthetic electron flow in Arabidopsis thaliana. J Biol Chem. 278(33):31286-31289

Whelan S, Goldman N. 2001. A general empirical model of protein evolution derived from multiple protein families using a maximumlikelihood approach. Mol Biol Evol. 18(5):691-699.

Wisecaver JH, Brosnahan ML, Hackett JD. 2013. Horizontal gene transfer is a significant driver of gene innovation in dinoflagellates. Genome Biol Evol. 5(12):2368-2381.

Wood PM. 1978. Interchangeable copper and iron proteins in algal photosynthesis: studies on plastocyanin and cytochrome c-552 in Chlamydomonas. Eur J Biochem. 87(1):9-19.

Worrall JAR, et al. 2007. Modulation of heme redox potential in the cytochrome $c_{6}$ family. J Am Chem Soc. 129(30):9468-9475.

Yoon HS, Hackett JD, Pinto G, Bhattacharya D. 2002. The single, ancient origin of chromist plastids. J Phycol. 38:40-40.

Yoon HS, Reyes-Prieto A, Melkonian M, Bhattacharya D. 2006. Minimal plastid genome evolution in the Paulinella endosymbiont. Curr Biol. 16(17):R670-672.

Zatwarnicki P, et al. 2014. Cytochrome $C_{6 B}$ of Synechococcus sp. WH 8102 - crystal structure and basic properties of novel $c_{6}$-like family representative. Biochem Biophys Res Commun. 443(4):1131-1135.

Associate editor: Rebecca Zufall 